Gabarda, S., Orellana Alonso, N. y Pérez Carbonell, A. (2017). La comunicación adolescente en el mundo virtual: una experiencia de investigación educativa. Revista de Investigación Educativa, 35(1), 251-267

DOI: http://dx.doi.org/10.6018/rie.35.1.251171

\title{
La comunicación adolescente en el mundo virtual: Una experiencia de investigación educativa
}

\author{
Adolescent Communication in the virtual Word: \\ An experience in educational research
}

\author{
Sara Gabarda, Natividad Orellana Alonso y Amparo Pérez Carbonell \\ Universitat de Valéncia
}

\begin{abstract}
Resumen
En este trabajo presentamos una experiencia de investigación educativa llevada a cabo en un centro de Educación Secundaria Obligatoria (ESO), cuya finalidad es conocer las problemáticas derivadas de la comunicación entre el alumnado del primer ciclo de secundaria dentro del mundo virtual, así como mejorar la calidad de vida de las personas involucradas en dicha problemática.

El diseño del proceso de investigación empleado es emergente y su metodología mixta. El curso y necesidades de la investigación nos llevaron a comenzar un proceso de investigaciónacción participante, en la que el propio alumnado y sus familiares formaron parte del proceso de cambio de las situaciones de riesgo a las que se enfrentan.

Los resultados obtenidos en esta investigación muestran que en este centro educativo requieren de un proyecto de sensibilización del uso saludable de internet. En el cual la participación activa de toda la comunidad educativa es un pilar fundamental.

Palabras clave: mundo virtual; comunicación; adolescencia y pensamiento crítico.
\end{abstract}

Correspondencia: Natividad Orellana, natividad.orellana@uv.es, Departamento de Métodos de Investigación y Diagnóstico en Educación, Facultad de Filosofía y Ciencias de la Educación, Av. Blasco Ibáñez, 30, 46010. Universitat de València (Valencia). 


\begin{abstract}
In this project, we present an educational research experience carried out in a compulsory secondary education center (ESO), whose purpose is to understand the problems derived from the communication among the first-year students of secondary education within the virtual world, as well as to improve the quality of life of the people involved in said problem.

The design of the research process employed is emerging and the methodology is mixed. The course and needs of the research led us to begin a process of participatory action research, in which the students themselves and their families were part of the process of changing the risk situations they face.

The obtained results in this research show that this educational center requires a project to raise awareness of healthy internet usage where the active participation of the entire educational community is essential.
\end{abstract}

Keywords: virtual world; communication; adolescent and critical thinking.

\title{
Introducción
}

Según Zygmunt Bauman (2008) vivimos en una modernidad que adjetiva como líquida, en la que todo cambia y evoluciona constantemente, en la que difícilmente podemos hacer planes a largo plazo y en la que algunos valores como el «compromiso» han cambiado completamente su sentido. En esta modernidad cambiante, el impacto de las tecnologías digitales es uno de los aceleradores clave en estas constantes modificaciones sociales. Por ello, las educadoras y educadores nos encontramos frente al enorme reto de preparar a las próximas generaciones para un mundo nuevo, cambiante y sobresaturado de información. Además, su abordaje debe comenzar por la aceptación del proceso de «fusión» social que estamos viviendo y proseguir con el aprendizaje de vivir y convivir saludablemente tanto en el mundo «online» como en el «offline».

Al tratar de conectar las características de esta era digitalizada, conectada y líquida con la etapa vital que nos ocupa, la adolescencia, pueden surgirnos las siguientes preguntas: ¿cómo afecta esto a las y los adolescentes?, ¿cómo repercute el cambio constante en la configuración de su identidad? y ¿cómo afecta a su relación con los demás?

\section{Identidad digital}

Bauman (2007) señala que para las y los jóvenes de hoy en día no es tan importante «la construcción de la identidad» como el hecho de tener la habilidad de reconstruirla siempre que tengan necesidad de ello, es decir de reidentificarse. Por otro lado, Reig (2013) también nos habla de la posibilidad que proporcionan las redes de reinventarnos a través de la creación de nuevas identidades, matizando que esto puede ser positivo si su resultado es la proyección de una imagen más compleja de nosotras y nosotros mismos.

La imagen que las y los adolescentes transmiten en las redes sociales es una combinación de aquello que son y lo que les gustaría llegar a ser. Una integración de las informaciones personales que comparten en diferentes contextos en el mundo no virtual, como por ejemplo, pensamientos privados, imágenes propias, noticias o gru- 
pos por los que siente interés, relatos sobre su vida cotidiana confeccionando todas estas informaciones una identidad digital que forma parte de la identidad individual y probablemente temporal de la o el adolescente. Algunos autores (Fairweather, 2010; McKenn \& Bargh, 2004; Valkengur \& Peter, 2010) destacan ciertos beneficios que la era digitalizada puede ofrecer tanto a la generación de la identidad como al proceso socializador en la adolescencia:

- Ayuda a construir la identidad deseada porque evita ciertos prejuicios que se producen en el contacto cara a cara.

- Genera un efecto positivo sobre determinadas competencias sociales de aquellas personas que son más tímidas o retraídas.

- Permite expresarse a través de otros medios y de manera más creativa (por ejemplo, a través de videos y fotografías) lo cual beneficia a aquellas personas que tienen más facilidad para comunicarse a través de estos medios.

- Aumenta el respeto a la diversidad. En muchas ocasiones el rechazo hacia las personas que son diferentes, proviene del miedo a lo desconocido. Las redes sociales permiten estar en contacto e interactuar con gente de diferentes culturas, capacidades, religiones, etc. y por tanto, rompe con ese desconocimiento.

Sin embargo, desde la literatura científica especializada también se está dando la voz de alarma sobre diferentes problemáticas que se están produciendo en este nuevo medio socializador, como es el caso del «ciberbullying». Un tipo de acoso que se ejerce a través de internet y que se produce entre escolares (Smith et al., 2008) y también puede darse en relaciones de noviazgo entre jóvenes (Durán \& Martínez-Pecino, 2014). Conectados con este fenómeno también se destacan otras problemáticas ejercidas bajo un contexto erótico-sentimental, como son: el «grooming», acoso ejercido por un adulto hacia un o una menor a través de internet (Pérez-San José et al, 2011); el «sexting», fenómeno referido a la difusión de contenidos de tipo sexual a través de internet (Mitchell, Finkelhor, Jones \& Wolak, 2012); y el «sextorsion», chantaje realizado a partir de la posesión de una imagen íntima de la víctima (Flores, 2010).

El engaño y falta de transparencia en la comunicación a través de internet, pueden producirse debido a las características intrínsecas de este medio y consecuentemente, fomentar las problemáticas mencionadas. No obstante, algunos autores como Back et al. (2010) afirman que, lo más habitual es que la interacción con otras personas en el mundo virtual dé lugar a vínculos de confianza y por tanto, esto genere una exigencia y responsabilidad de transparencia y veracidad entre unas personas y otras. Por otro lado, Stornaiuolo, Dizio y Hellmich (2012) en su estudio sobre las relaciones de los adolescentes en comunidades virtuales también señalan que los jóvenes se comunican con cautela en sus primeras interacciones con personas desconocidas e intentan confirmar la veracidad de las afirmaciones del otro. Si se encuentran en un entorno de seguridad comparten más información real con los demás y tratan de vincularse a la comunidad virtual de la que forman parte.

Como acabamos de exponer, la llegada de la Web 2.0 ha supuesto una revolución en la forma de relacionarnos y comunicarnos entre la población juvenil, así como en la manera de gestionar su privacidad e identidad. 


\section{El papel de la educación}

Para que el intercambio social en la red tenga connotaciones positivas debe estar acompañado y guiado de una educación y unos valores adecuados. Por ejemplo, es importante que las y los adolescentes conozcan y reflexionen sobre: las características intrínsecas de la red, las leyes de privacidad que aceptan al entrar en determinadas redes sociales, cómo decidir con quién compartir su información personal y las repercusiones que ello conlleva, normas de comportamiento en la comunidad virtual, la importancia del respeto o la empatía hacia los demás a la hora de interactuar en la red, entre otros.

A la hora de activar procesos de prevención cabe tener presente que la educación basada en el control y restricción carece de sentido en un mundo virtual en el que todo cambia y evoluciona constantemente. Por este motivo, aprender a utilizar las tecnologías de la información y comunicación (TIC) de forma responsable y crítica, potenciar los beneficios que genera y amainar los riesgos, amenazas y efectos negativos asociados a estas, resulta la opción más coherente en la educación de las y los menores a largo plazo. Freire (1992-2002) pensaba que las personas no somos seres de «adaptación» sino de «transformación» y por ello, concibió el proceso de alfabetización como un poderoso instrumento de empoderamiento y transformación social. En el trabajo realizado; aplicamos esta misma idea al proceso de alfabetización digital, el cual, debe ir más allá de la mera adquisición de habilidades de uso y servir como medio de desarrollo del espíritu crítico y del empoderamiento al que Freire apelaba.

Para conseguir que este reto educativo sea posible es necesaria la suma de sinergias y la implicación de todos los agentes educativos: estudiantes, centros educativos, familiares y el resto de la comunidad educativa.

En el capítulo 3, artículo 9 de la Ley 26/2015, de modificación del sistema de protección a la infancia y a la adolescencia se señala que:

A través del sistema educativo se implantará el conocimiento que los menores deben tener de sus derechos y deberes como ciudadanos, incluyendo entre los mismos aquellos que se generen como consecuencia de la utilización en el entorno docente de las Tecnologías de la Información y Comunicación.

Para llevar esta tarea a cabo es necesario que los centros educativos complementen sus programas de convivencia con intervenciones dedicadas a las relaciones en el mundo virtual. Así como también, es necesario acciones de sensibilización y formación en el uso responsable de las redes sociales, implicando tanto a las familias como al profesorado de los centros y promoviendo el protagonismo y la participación activa de los y las jóvenes en estas acciones (Tejedor \& Pulido, 2012).

Las tareas desarrolladas desde los centros educativos son fundamentales, pero carecen de sentido si no se llevan a cabo con la colaboración de las familias, ya que estas son un agente educativo y socializador fundamental. Los espacios tradicionales de socialización en las familias se están ampliando y las personas que interactúan con sus hijos e hijas a través del mundo virtual ejercen una fuerte influencia en el establecimiento de normas de comportamiento en la comunidad y objetivos de aprendizaje. Al mismo tiempo, las y los menores adquieren el rol de enseñar a sus familiares el 
manejo de estas TIC. Se produce así, una reducción de la brecha digital existente entre las diferentes generaciones de la familia y se genera la posibilidad de que todas las personas puedan participar juntos de un proceso de aprendizaje y empoderamiento en el uso de estas nuevas herramientas comunicativas (Reig, 2013). Ejercer un control parental restrictivo, carece de sentido, ya que este no previene el mal uso de las TIC, al contrario, ya que en las familias con alta cohesión social y comunicación saludable estos riesgos se reducen (Sasson \& Mesch, 2014).

Además del personal docente y las familias, el resto de la comunidad educativa también tenemos responsabilidades ciudadanas en la educación de las y los menores. Como señala Muñoz (2012),-es importante construir redes de convivencia que nos permitan adquirir nuestra responsabilidad ciudadana para mejorar nuestras relaciones cotidianas y evitar así cualquier tipo de violencia injustificada. Nuestro ejemplo ๑tanto dentro como fuera de la red- es fundamental para que la población infantil y juvenil tenga referencias de cómo relacionarse con los demás de manera sana. Por ello, cabría preguntarnos si nosotras y nosotros mismos cumplimos las normas de convivencia virtual que les estamos reclamando: ¿excluimos deliberadamente a personas de grupos de conversación?, ¿pensamos dos veces antes de colgar determinados comentarios o fotos en la red? o ¿toleramos o denunciamos los comentarios violentos que encontramos en la red? La ciberconvivencia es una de nuestras tareas pendientes y la mejora de esta, seguramente reduciría la conflictividad negativa y las situaciones de riesgo a través de internet. Adaptando las palabras de Gandhi al tema que nos ocupa, seamos el cambio que queremos ver en nuestras y nuestros adolescentes.

\section{Método}

\section{Objetivo}

Exponer la experiencia de investigación educativa seguida para dar respuesta a la preocupación de toda una comunidad educativa en lo referente a las formas de relación y comunicación a través del mundo virtual que el alumnado adolescente utiliza. Planteamiento que hace visible la necesidad del reconocimiento de riesgos en los nuevos espacios de intercambio social en la red para actuar de manera preventiva sobre ellos.

\section{Población y Muestra}

Dadas las características de este estudio y que su finalidad no es de carácter inferencial, en este subapartado haremos referencia a los implicados en esta investigaciónacción participante.

Los participantes en este estudio son el alumnado de primer ciclo de la ESO (160 estudiantes) de un instituto público de ubicado en la Comunidad Valencia, diferentes miembros de la comunidad educativa a la que pertenecen y distintos especialistas en mediación y ciberconvivencia. Los miembros de la comunidad educativa involucrados en este proyecto -realizado durante el año académico 2014- son: familiares del alumnado, el director, el jefe de estudios, 7 tutoras y un tutor de los grupos de $1^{\mathrm{o}}$ y $2^{\mathrm{o}}$ de la ESO del centro. Y por último, los especialistas participantes en el proyecto son: un 
mediador y una mediadora escolar, un policía local responsable de la prevención de acoso escolar y dos formadores de formadores en el uso responsable de las TIC.

\section{Instrumento}

Tal y como podemos ver en la figura 1, los instrumentos empleados en la recogida de datos fueron: diario de campo, cuestionarios, entrevistas consultivas y grupos debate.

\begin{tabular}{|c|c|c|c|}
\hline TÉCNICA & OBSERVACIÓN & ENCUESTA & TÉCNICAS DE GRUPO \\
\hline INSTRUMENTO & DIARIO DE CAMPO & $\begin{array}{c}\text { ENTREVISTAS } \\
\text { CONSULTIVAS }\end{array}$ & CUESTIONARIOS \\
\hline $\begin{array}{c}\text { MOMENTOS DE } \\
\text { USO }\end{array}$ & $\begin{array}{c}\text { DURANTE TODO EL } \\
\text { PROCESO DE LA } \\
\text { INVESTIGACIÓN }\end{array}$ & $\begin{array}{c}\text { PRIMERAS FASES DE LA } \\
\text { INVESTIGACIÓN. }\end{array}$ & $\begin{array}{c}\text { ÚLTIMA FASE DE LA } \\
\text { INVESTIGACIÓN. }\end{array}$ \\
\hline
\end{tabular}

Figura 1. Técnicas e instrumentos empleados

\section{Procedimiento de recogida datos}

El diseño de la investigación es de tipo emergente, y comenzó con el planteamiento de una pregunta específica «¿el alumnado del centro se relaciona de manera saludable a través del mundo virtual?» y a partir de esta, se fueron generando nuevas preguntas que fueron guiando todo el proceso de investigación.

En su respuesta recurrimos a técnicas que fomentan el pensamiento divergente y creativo; con el fin de evitar bloqueos, ampliar el abanico de posibilidades tanto en el proceso de indagación como en la propuestas de soluciones (¿qué personas pueden enriquecer la investigación?, ¿qué alternativas podemos seguir para obtener información de difícil acceso?, ¿qué recursos pueden despertar un mayor interés para la comunidad educativa?, ¿cómo podemos mejorar la realidad del alumnado del centro educativo?...). Para ello, empleamos como referencia la guía Design Thinking for Educators de IDEO (2012), que junto con la observación sistemática y controlada fue una técnica crucial en el proceso de recogida y contextualización de la información.

Con el fin de clarificar el proceso seguido, a continuación presentamos la siguiente figura -ver figura $2-$.

1. Fase 1: relevancia

A través de esta fase se persiguió conocer y contrastar el interés y perspectiva de diferentes agentes sobre el tema investigado, para poder confirmar así, la relevancia de continuar con el proceso de indagación iniciado.

Para tal fin, recogimos información del equipo directivo y de profesionales expertos externos a través de 4 entrevistas abiertas y con finalidad consultiva, a través de las cuales se consultó acerca de sus conocimientos, experiencias profesionales y reflexiones en relación con el tema investigado. Estos datos se complementaron con el pase de un breve cuestionario a 43 familiares del alumnado del centro en el que se preguntó sobre las redes sociales que utilizan habitualmente, las redes que emplean los y las menores 


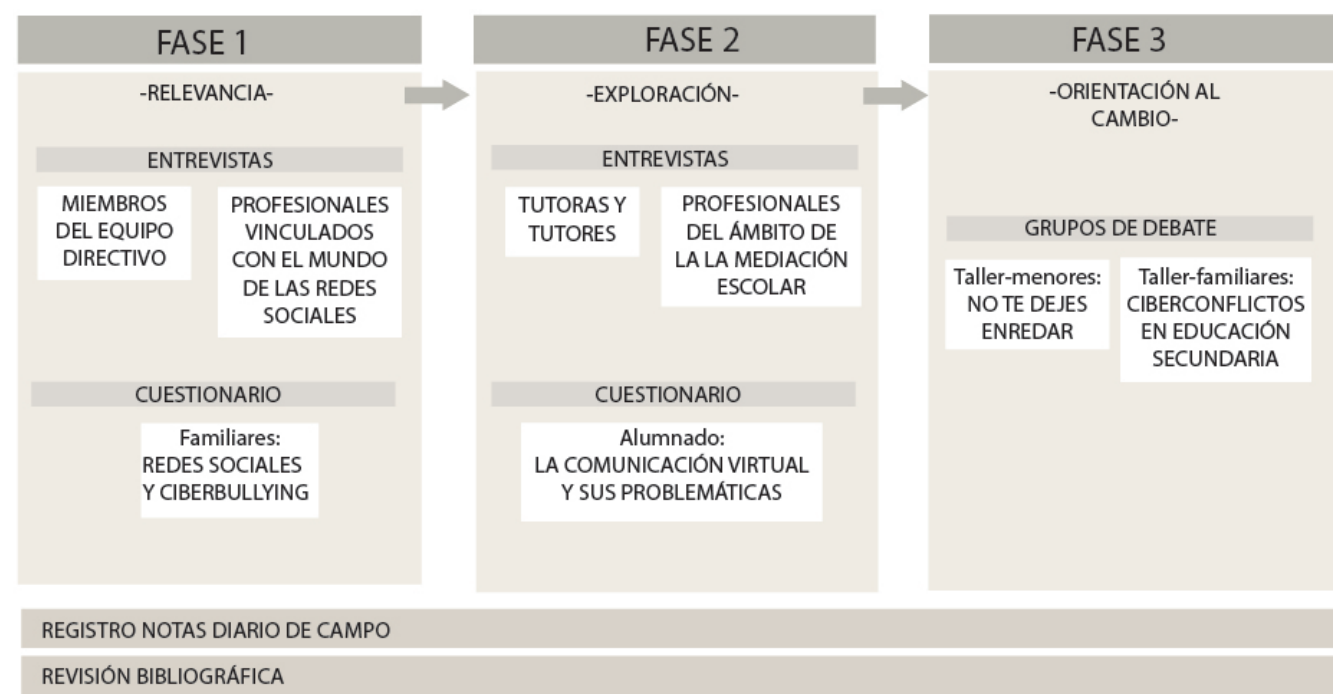

Figura 2. Fases del proceso de investigación

a su cargo y su interés en ampliar su formación en la comunicación y problemas de acoso en el mundo virtual adolescente. Tras contrastar esta información concluimos que «sí» era relevante continuar con este proceso de investigación, lo que nos llevó a profundizar en la consulta y revisión bibliográfica de literatura especializada en este tema.

2. Fase 2: exploración

Con esta segunda fase buscamos conocer datos concretos sobre la realidad del alumnado del centro. Para ello, se pasó un cuestionario -creado para el presente estudio- a un grupo de 160 alumnos y alumnas del primer ciclo de secundaria en los que se recogió información acerca del uso de las redes sociales y las problemáticas derivadas del uso de estas (redes sociales que utilizan, dispositivos tecnológicos que poseen, conflictividad a través de la red, reacción ante casos de acoso entre compañeros/as, control parental y personas a las que acudir ante los conflictos). La finalidad del mismo era conocer la situación concreta de este alumnado con el que posteriormente se iba a intervenir.

Esta información se complementó con 2 entrevistas abiertas y de carácter consultivo a 2 tutoras y a 3 profesionales en el ámbito de la mediación escolar. Al igual que en las entrevistas realizadas previamente, se les consultó acerca de sus conocimientos, experiencias profesionales y reflexiones en relación con el tema investigado.

3. Fase 3: orientación al cambio:

Puesto que pretendemos que las y los adolescentes y sus familiares sean partícipes activos de este proceso de indagación educativa, a partir de esta fase apostamos por emplear un diseño de investigación orientado al cambio, concretamente con el modelo de investigación-acción participativa. 
Kemmis y McTaggart (2005) señalan que este modelo se caracteriza por la propiedad compartida de los proyectos de investigación, el análisis de problemas sociales basados en la comunidad y una orientación hacia la acción comunitaria. Además, tienen un carácter cíclico en forma de espiral con una serie de momentos sucesivos en cada uno de sus ciclos: planificación, acción-observación y reflexión. Debido a las limitaciones espaciotemporales del estudio, únicamente se llevó a la práctica un primer ciclo de este proceso.

a) Planificación: en este momento del ciclo, y a partir de los datos recogidos en fases anteriores, planificamos qué contenidos se trabajarían en los talleres a los que asistiría el alumnado y sus familias. Para ello, atendimos a las problemáticas que con mayor frecuencia se reflejaban en la información que las audiencias nos habían facilitado: ciberbullying, malentendidos en la red, ciberconflictos, sexting, sextorsión y grooming. Y finalmente, confeccionamos diferentes listados de recursos que invitaban a la reflexión e intercambio de opiniones durante el desarrollo de las diferentes actividades, todos estos adaptados al contexto y realidad de cada uno de los grupos.

b) Observación-Acción: la puesta en práctica de este momento del proceso se llevó a cabo a través de dos talleres, en los que la participación fue de carácter voluntario:

- No te dejes Enredar: taller dirigido al alumnado, en el que participaron 16 alumnas y 9 alumnos en 3 sesiones diferentes.

A lo largo de estas sesiones se realizaron diferentes actividades generadoras de debate grupal (video-fórum, role-playing y paneles de lluvia de ideas, entre otras) a través de las cuales se trabajaron los siguientes temas: ventajas y desventajas del uso de las TIC, recursos y análisis del lenguaje en las redes sociales, conflictos en la red y conductas de acoso ejercidas a través de la red (ciberbullying, grooming y sexting).

- Ciberconflictos en Educación Secundaria: taller dirigido a los familiares del alumnado del centro, en el que participaron un total de 10 madres organizado en 1 sesión.

A través de esta sesión se activó un debate grupal generado a partir de una actividad de -verdadero o falso- basada en el artículo Cyberbullying mitos y realidades de Sabella, Patchin y Hinduja (2013). Los temas tratados a través de este recurso fueron: diferencias conceptuales entre ciberconflictos y situaciones de acoso online, tipologías y ejemplos de ciberconflictos, control parental e intervención preventiva.

Con el fin de preservar el respeto hacia y entre las personas participantes de ambos talleres y de revisar la propia práctica educativa, se acordaron los siguientes 3 principios:

- Respetar la libre expresión durante los debates, siempre y cuando estas intervenciones no atentarán contra la integridad moral de otras personas.

- La recogida de datos, como parte del proceso de investigación siempre irá supeditada al respeto, la dignidad y el derecho a la privacidad de las personas participantes. 
- Dar opción y potenciar que todas las voces puedan participar en las intervenciones que se generen a través del diálogo igualitario.

c) Reflexión: tras finalizar los talleres se llevó a cabo un proceso de reflexión sobre todos los temas tratados, seguido de propuestas de acción para cambiar todo aquello que afecta negativamente a la ciberconvivencia de las personas miembro de la comunidad educativa, especialmente a la del alumnado. Este último momento del proceso de investigación-acción también sirvió para evaluar y analizar la propia práctica educativa.

\section{Análisis de datos}

El análisis de los datos cuantitativos se realizó con el programa SPSS-22. Se emplearon descriptivos y tablas cruzadas con la finalidad de tener una base para triangular toda la información recopilada y en ningún caso con finalidad inferencial.

Para el análisis de los datos cualitativos se tomó como referencia la técnica analítica que Glaser \& Strauss (1967) denominaron como el "Método de Comparación Constante". Este método propicia la creación de un sistema de organización de los datos a través de un proceso inductivo, generativo y constructivo y permite contrastar información recogida desde diferentes instrumentos, lo cual se adecua a las características de este proceso de investigación.

De este modo, se plantea un análisis caracterizado por la triangulación de información, a través del cual se complementa la información obtenida de instrumentos de recogida de información de vertiente metodológica cuantitativa y cualitativa. Para posteriormente, contrastar todos estos datos con la literatura especializada y con las conclusiones extraídas de los propios participantes en la fase de investigación-acción.

\section{Resultados}

A continuación presentamos los resultados de manera secuencial, tomando como secuencia en su exposición las diferentes fases de nuestro proceso de indagación.

\section{Entrevistas y registro de notas de campo}

De las entrevistas y del registro de notas de campo realizadas a miembros del equipo directivo, profesorado, expertos en mediación escolar y usos de las redes sociales se recogieron las siguientes opiniones coincidentes:

- Se producen conflictos y agresiones entre el alumnado del centro educativo, tanto de manera online como offline.

- La implicación de las familias en los proyectos educativos propuestos por el centro es fundamental. No obstante, es muy complicado activar esta participación.

- El profesorado requiere de mayor información y formación en el uso responsable de las nuevas tecnologías de la información y comunicación.

- Es necesario desarrollar protocolos de actuación de centro en el que se especifique cómo intervenir ante situaciones de riesgo por parte del alumnado en la red. 
Esta información fue la empleada para la planificación de los talleres, así como para la confección de conclusiones y recomendaciones dadas al centro educativo.

\section{Cuestionario}

- Cuestionario (familiares) "Redes sociales y ciberbullying":

Tal y como adelantábamos en el apartado anterior, la finalidad de este cuestionario fue, principalmente, conocer la relevancia que suponía este tema para las familias como parte de la comunidad educativa. De los resultados obtenidos, cabe resaltar que el $81.4 \%$ de las personas encuestadas señaló que era usuaria de alguna red social y el $74.4 \%$ nos facilitaron su contacto porque les gustaría tener más información acerca de las características de la comunicación online en la adolescencia y las posibles problemáticas que pueden producirse derivadas de su mal uso.

- Cuestionario (Alumnado) "La comunicación virtual y sus problemáticas":

Del total del alumnado encuestado, el $91.9 \%$ dispone de ordenador y/o de móvil y el $98.7 \%$ se comunica a través de alguna red social virtual, siendo WhatsApp $(87.5 \%)$ la red social que más estudiantes utilizan, seguida de Facebook $(56.2 \%)$, Tuenti (42.5\%), Twitter (40.6\%) e Instagram (33.8\%).

En cuanto a las situaciones conflictivas a través de Internet -ver figura 3-, más de la mitad del alumnado (59.5\%) afirma haber tenido conflictos a través de las redes y el $41.6 \%$ de estos se han producido como consecuencia de un conflicto ocurrido con anterioridad de manera offline. Por otro lado, más de la mitad de las y los jóvenes $(58.7 \%)$ ha sido testigo de una situación de hostigamiento hacía otro menor y un $36.3 \%$ de estos indica que su reacción fue ofrecerle ayuda a ese compañero o compañera. El 26.9\% del alumnado atestiguó «haber sido molestado» a través de internet, por otro igual, «a veces». Esta cifra se reduce al 5.0\% en los casos en los que el hostigamiento se ha producido «muchas veces». Estos datos se complementan con el $19.4 \%$ del alumnado que afirma haber agredido o molestado de manera online a otro menor, «a veces» y el 5.0\% que señala haberlo hecho en «muchas veces».

Fijándonos en la variable de género -ver figuras 4 y 5-, encontramos que las chicas son las que más problemas tienen a través de las redes, ya que señalan «haber molestado» «a veces» $\mathrm{y}$ «haber sido molestadas» tanto «a veces» como «muchas veces» en mayor porcentaje que los chicos. En el caso de «haber molestado» «muchas veces» el porcentaje de chicos y chicas es similar.

Por último, resaltar que el 50.1\% del alumnado afirma que si se encontrara ante una situación de riesgo en internet lo compartiría con una persona adulta -ver figura $6-$, un $42.8 \%$ solo se lo contaría a otro menor y el $7.1 \%$ no se lo contaría a nadie. Este último caso es el más preocupante, ya que dificulta realizar una detección precoz de la situación en la que se encuentran. 


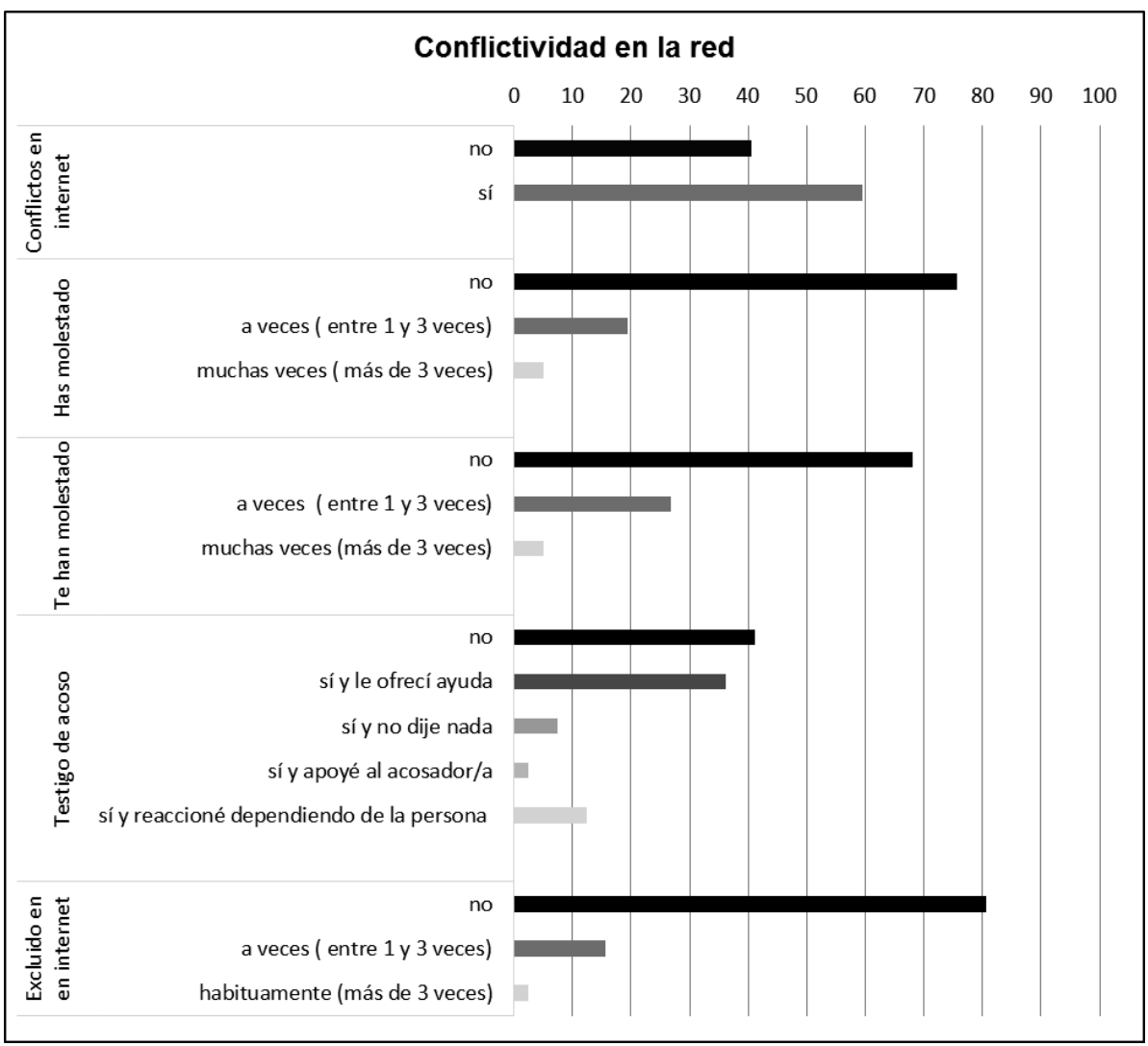

Figura 3. Conflictividad en la red

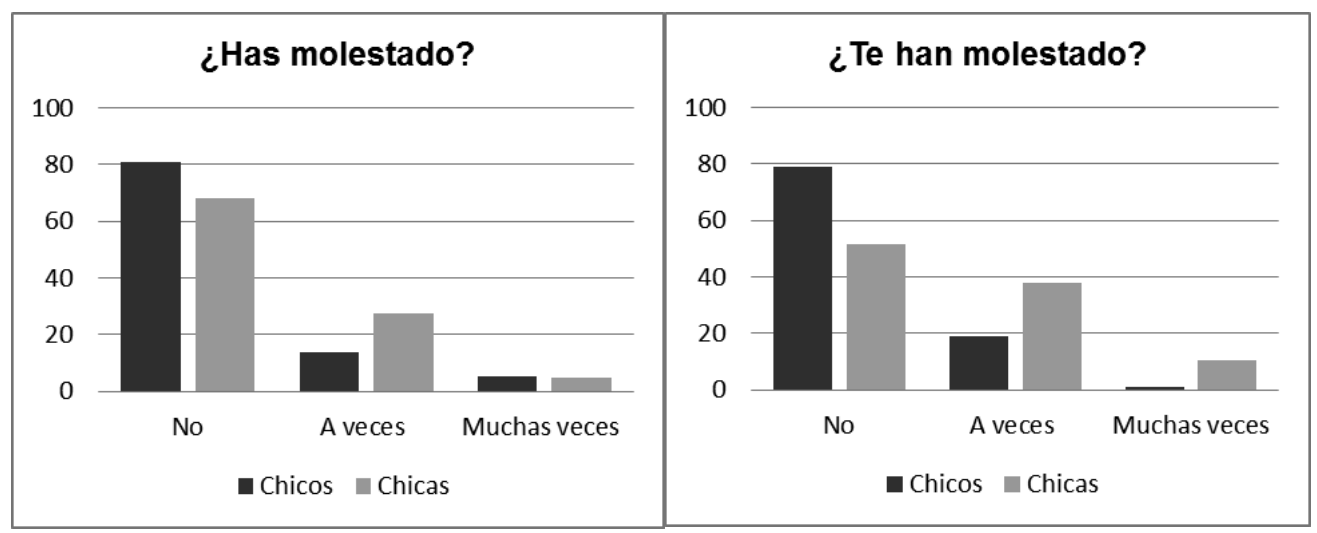

Figuras 4 y 5. Porcentajes de Chicos y Chicas que «ha molestado» y que «han sido molestada» través de la red. Escala: no, a veces (entre 1 y 3 veces), y muchas veces (más de 3 veces) 


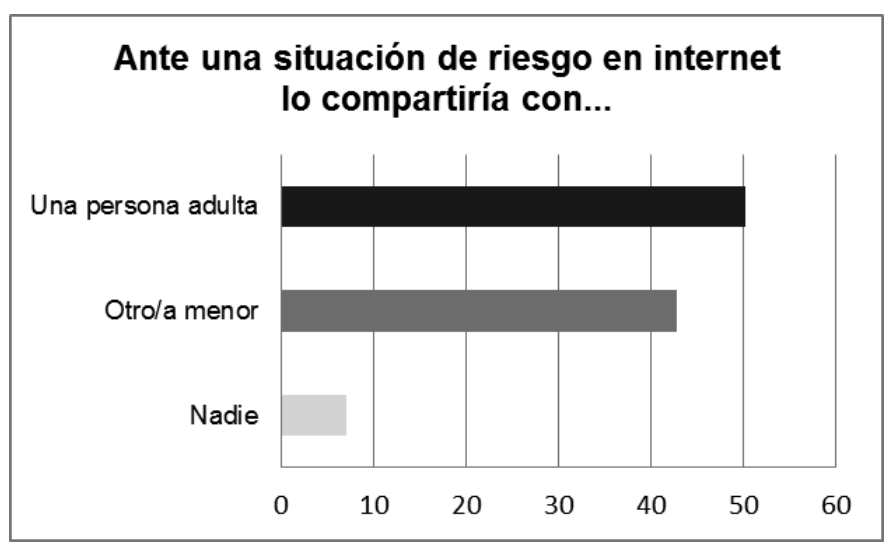

Figura 6. Variable «Personas de apoyo: ¿con quién compartirías una situación de acoso?»

\section{Grupos debate}

Los grupos debate se realizaron con alumnado del primer ciclo de secundaria y con madres del centro educativo, en los dos talleres que anteriormente explicados en la fase 3.

- Taller no te dejes enredar:

A través de este taller el alumnado compartió experiencias y conocimientos sobre la convivencia a través de internet y los peligros con los que pueden encontrarse derivados por su mal uso. Todo el alumnado demostró tener un claro dominio del lenguaje propio de las redes sociales (tanto como emisores como receptores). La mayoría del alumnado encuentra más aspectos positivos que negativos a la interacción a través del mundo virtual. Siendo las ventajas más señaladas «ver las fotos y los videos de otras personas» $\mathrm{y}$ «quedar con tus amigos y amigas» y la desventaja más destacada, «que te hable un desconocido o alguien que no quieras».

Pese a estos resultados, el 100\% afirmó haber tenido conflictos a través de las redes sociales y atestiguaron conocer casos ciberbullying, grooming y sexting, que se habían producido en su entorno próximo. Pese a que 5 participantes compartieron experiencias positivas al compartir con sus familiares conflictos vividos en la red, otras 3 menores destacaron el miedo a que sus familiares las culpabilizaran, al encontrarse en una situación en internet comprometida, y a que reaccionaran restringiéndoles su uso. También resaltaron el miedo al reproche por parte de sus familiares y amistades. «Te dije hace muchísimo tiempo que no lo hicieras y a ti te dio igual, te advertí que no hablaras con gente que no conoces por Internet», es un ejemplo de cómo verbalizó una menor esta sensación a través de una actividad de role-playing.

Entre las propuestas que hicieron para mejorar la comunicación y para evitar peligros en la red, «crear una red social para ayudarse entre el alumnado del centro ante situaciones de peligro o problemas de comunicación» fue la más resaltada. 
- Ciberconflictos en educación secundaria:

El 100\% de las madres que formaron parte del taller son usuarias de Whatsapp y el 70\% de ellas también utilizan Facebook, coincidiendo estas con las más utilizadas también entre el alumnado de primer ciclo de ESO. Las participantes afirmaron ser conocedoras de algunos de los conflictos y problemáticas que se han producido en el centro educativo a través del mundo virtual. En su totalidad piensan que el control parental en el uso de internet es necesario, siendo el diálogo familiar el mejor medio para llevarlo a cabo y que factores como la edad de los y las adolescentes y el desarrollo de las TIC influyen en el tipo de medidas de control que los padres y madres utilizan. También destacar que algunas madres reconocen que las personas adultas no siempre hacemos un uso adecuado de las TIC y que por ello, también requerimos de formación. En cuanto a la prevención de problemáticas en la red, opinan que es necesario ofrecer un ambiente seguro y de confianza, así como estar atentas a los cambios anímicos de sus hijos e hijas y estar informadas sobre todo lo que rodea al mundo de las tecnologías digitales. En este tema también señalan que ante una situación de violencia escolar es importante proteger tanto a la víctima como a la persona que ha errado, ya que ambos necesitan apoyo para superar dicha situación.

Como propuestas para la prevención de problemáticas en la red, propusieron más formación para el alumnado y sus familias desde el centro educativo. Aunque reconocen los potenciales problemas que puede presentar el uso de internet, las participantes se mostraron positivas frente al uso del mismo.

\section{Discusión y conclusiones}

La revolución tecnológica y la velocidad a la que se producen sus avances han hecho que la forma de relacionarnos, comunicarnos y la manera de gestionar nuestra privacidad e identidad cambien a gran velocidad y de manera constante. Que las y los jóvenes se beneficien o no de este nuevo mundo virtual depende del uso que se le dé a estas herramientas tecnológicas. Para conseguir este buen uso social de internet es necesaria la educación, y para ello, es necesario conocer las potencialidades educativas y sociales de las tecnologías digitales (Cabra \& Marciales, 2012). El mundo virtual es una realidad que cambia constantemente. Por tanto, la educación basada en el control y restricción del uso de estas tecnologías carece de sentido y resulta más efectivo apostar por una educación a largo plazo a través de la cual aprendan a utilizar las TIC de forma responsable y crítica, potenciar los beneficios que generan y empoderarse frente a las posibles amenazas que encuentren con el uso de estas.

A la vista de estos resultados, es necesario reparar en la forma en la que las y los jóvenes se relacionan y comunican a través de internet y llevar a cabo acciones dirigidas a la prevención e intervención de problemáticas producidas en la misma. Sobre todo, poniendo especial atención en las chicas, ya que en nuestro estudio se refleja que son las que se ven más involucradas en situaciones conflictivas, tanto como agresoras como víctimas -especialmente ejerciendo este último rol-. Contrastando este resultado con literatura especializada en acoso escolar a través de internet, encontramos que no existe un consenso claro sobre la influencia de la variable del género; mientras que algunos trabajos señalan que las chicas asumen este rol predominantemente como 
víctimas (Calmaestra, 2011 y Félix-Mateo, Soriano Ferrer, Godoy Mesas \& Sancho Vicente, 2010), otros no destacan diferencias significativas (Álvarez García et al., 2011 y García-Fernández, 2013). También resaltar que la mayoría de casos de conflictos o situaciones violentas a través de internet se han producido dentro de un contexto erótico-sentimental; pudiéndose incluso clasificar algunos casos como situaciones de sexting y grooming. Por ello, también es necesario trabajar la prevención y sensibilización de la violencia bajo este contexto, así como es necesario ampliar los estudios de investigación acerca de esta variable.

El control parental abordado desde una relación de confianza entre el estudiantado y sus familiares es muy importante dentro del trabajo de prevención y detección de problemáticas en la red (Sasson \& Mesch, 2014). Tal y como explicaron las madres participantes en el taller, este control debe adecuarse a la edad y usos de las TIC de los y las menores. Además, es muy importante evitar juzgarles o culpabilizarles cuando están sufriendo cualquier tipo de agresión a través de internet (Save the Children, 2014), ya que tal y como expusieron algunas de las menores en el taller, este es uno de los motivos por los que son reacias a compartir con otras personas este tipo de problemática. En relación con la implicación familiar, también hay que destacar que aunque el $81.4 \%$ de los padre y madres encuestados indicaron estar interesados en aprender más sobre esta tema, únicamente asistieron 10 madres al taller orientado a las familias. Por lo cual, sería interesante profundizar en los motivos que provocan que la participación e implicación parental en los centros educativos de educación secundaria sea tan escasa y que predominantemente sean las madres las que asumen esta responsabilidad.

En cuanto al papel de los centros educativos, los diferentes miembros de la comunidad educativa coinciden con la literatura científica especializada (Gómez-Ortíz, Del Rey, Casas \& Ortega-Ruiz, 2014) en que es necesaria la formación y sensibilización en el uso responsable de las TIC. Así como también se requiere mejorar la organización de los centros educativos, implementado acciones relacionadas con el uso saludable de las TIC en su Plan de Convivencia de Centro y confeccionando protocolos de actuación para situaciones de cualquier tipo de violencia entre el alumnado a través de Internet. Ya que como señala Garaigordobil (2015) aunque en los últimos años ha aumentado considerablemente la sensibilización sobre la importancia de trabajar la (ciber)convivencia en los centros escolares, la aplicación sistemática de programas específicos sobre esta temática sigue siendo una de nuestras asignaturas pendiente.

Tal y como indica Muñoz (2012), es necesario que toda la comunidad se implique para construir redes de convivencia que fomenten la responsabilidad de la ciudadanía, y poder así, resolver los conflictos generados en nuestras relaciones cotidianas. Para llevar esto a cabo es importante que todas las personas aprendamos a respetarnos siguiendo normas de convivencia tanto dentro como fuera de la red. Creando así, con nuestras acciones un entorno seguro y respetuoso para todas las personas en el mundo online.

\section{Limitaciones y perspectiva de futuro}

En esta investigación, la participación de diferentes miembros de la comunidad educativa ha sido clave para poder obtener una visión global y compleja de la reali- 
dad estudiada. No obstante, no conseguimos toda la representatividad y participación deseada. El caso más significativo fue la escasa y sesgada participación en el taller dirigido a familiares del alumnado. El calendario y horario académico del centro delimitó las fases, los tiempos y la puesta en práctica del proceso de investigación-acción. Otro factor a tener presente en esta investigación es la rapidez con la que avanzan y cambian de las TIC, lo cual provoca que cualquier información recogida pueda quedar obsoleta en un periodo de tiempo relativamente breve, como por ejemplo ocurre con los medios o redes sociales que el estudiantado emplea para comunicarse. A pesar de estas limitaciones, los resultados obtenidos y la evaluación del propio proceso de investigación sí que han hecho posible detectar cuáles son las necesidades prioritarias de esta comunidad educativa.

Con los resultados obtenidos esperamos contribuir a la construcción del conocimiento colectivo sobre el uso de los nuevos espacios de comunicación social, sus riesgos, potencialidades e incidir en la importancia de la investigación e intervención preventiva desde el ámbito educativo en este campo.

\section{Agradecimientos}

Para finalizar, agradecer a todas las personas que participaron directa o indirectamente en este proyecto cediéndonos su tiempo y en especial, al centro educativo en el que se realizó este estudio.

\section{Referencias}

Bauman, Z. (2007). Los retos de la educación en la modernidad líquida. Barcelona: Gedisa. Bauman, Z. (2008). Una nova escena del drama entre vell $i$ jove. Generalitat de Catalunya Departament d'Acció Social i Ciutadania Secretaria de Joventut.

Álvarez-García, D., Núñez, J. C., Álvarez-Pérez, L., Dobarro, A., Rodríguez, C., \& González-Castro, P. (2011). Violencia a través de las tecnologías de la información y la comunicación en estudiantes de secundaria. Anales de psicología, 27(1), 221-231. Recuperado de http://www.redalyc.org/articulo.oa?id=16717018026

Back, M. D., Stopfer, J. M., Vazire, S., Gaddis, S., Schmukle, S. C., Egloff, B., \& Gosling, S.D. (2010). Facebook profiles reflect actual personality, not self-idealization. Psychological Science, 21(3), 372-374. doi: 10.1177/0956797609360756

Bargh, J. A., \& Mckenna, K. Y. A. (2004). The Internet and social life. Annual Review of Psychology, 55, 573-590. doi: 10.1146/annurev.psych.55.090902.141922

Cabras, F., \& Marciales, G. (2012). Comunicación electrónica y cyberbullying: Temas emergentes para la investigación e intervención Socioeducativa. Psicología desde el Caribe, 29 (3), 707-730. Recuperado de http://www.redalyc.org/articulo.oa?id=21328599008

Calmaestra, J. (2011). Cyberbullying: Prevalencia y características de un nuevo tipo de bullying indirecto. (Tesis Doctoral, Universidad de Córdoba). Recuperado de http://helvia. uco.es/xmlui/handle/10396/5717

Durán, M., \& Martínez-Pecino, R. (2015). Ciberacoso mediante teléfono móvil e Internet en las relaciones de noviazgo entre jóvenes. Comunicar, 44 (XXII), 159-167. doi: http://dx.doi.org/10.3916/C44-2015-17. 
Fairweather, L. (2010). Psychology, meet YouTube. Recuperado de https://www.youtube. com/watch? $v=m Y Q d U 0 G 5 J s E$

Félix-Mateo, V., Soriano Ferrer, M., Godoy Mesas, C., \& Sancho Vicente, S. (2010). El ciberacoso en la enseñanza secundaria. Aula abierta, 38(1), 47-58. Recuperado de http://redined.mecd.gob.es/xmlui/handle/11162/5058

Flores, J. (2010). Sextorsión, prácticas arriesgadas y fallos de seguridad al servicio del delito. Pantallas amigas. Recuperado de http://www.pantallasamigas.net/proteccioninfancia-consejos-articulos/index.shtm\#1

Freire, P. (2002). Pedagogía de la esperanza (5ª edición). Madrid: Siglo XXI.

Garaigordobil, M. (2015). Ciberbullying en adolescentes y jóvenes del País Vasco: Cambios con la edad. Anales de psicología 21(3), 1069-1076. doi: http://dx.doi.org/10.6018/ analesps.31.3.179151

García-Fernández, C.M. (2013). Acoso y ciberacoso en escolares de primaria: factores de personalidad y de contexto entre iguales. (Tesis Doctoral, Universidad de Córdoba). Recuperado de http://helvia.uco.es/xmlui/handle/10396/10949

Glaser, B., \& Strauss, A. (1967). El desarrollo de la teoría fundada. Chicago, Illinois, EE.UU.: Aldine.

Gómez-Ortiz, O., Del Rey, R., Casas, J. A., \& Ortega-Ruiz, R. (2014).Estilos parentales e implicación en bullying .Cultura y Educación: Revista de teoría, investigación y práctica, 26(1), 145-158. doi: 10.1080/11356405.2014.908665

IDEO (2012). Design Thinking for Educators Toolkit. Recuperado de http://www.designthinkingforeducators.com/

Kemmis, S., \& McTaggart, R. (2005). Participatory Action Research: Communicative Action and the Public Sphere. En N. K. Denzil \& Y. S. Lincoln (Eds.). The Sage Handbook of qualitative research (3 $3^{\text {a }}$ edición, pp. 559-604). Thousand Oaks, California, EE.UU.: Sage.

Ley 26/2015, de 28 de julio, de modificación del sistema de protección a la infancia y a la adolescencia. BOE-A-2015-8470. Recuperado de https://www.boe.es/diario_boe/ txt.php?id=BOE-A-2015-8470

Mitchell, K. J., Finkelhor, D., Jones, L. M., \& Wolak, J. (2012). Prevalence and characteristics of youth sexting: a national study. Pediatrics, 129(1), 13-20. doi: 10.1542/ peds.2011-1730.

Muñoz, Y. (2012). La participación comunitaria para afrontar los conflictos locales. En C. Manzanos, (Coord.). Mediación y participación en conflictos para transformar la sociedad comunitaria, política, económica, familiar y penal. (pp. 31-35). País Vasco: IKUSBIDE.

Pérez-San José, P., Flores, J., De la Fuente, S., Álvarez-Alonso, E., García-Pérez, L., \& Gutiérrez, C. (2011). Guía sobre adolescencia y sexting: qué es y cómo prevenirlo. Observatorio de la seguridad de la información de Instituto Nacional de Tecnologías de la Comunicación Pantallas amigas. Recuperado de https://www.incibe.es/CERT/ guias_estudios/guias/Guia_sexting

Reig, D. (2013). Describiendo al hiperindividuo, el nuevo individuo conectado. Los jóvenes en la era de la hiperconectividad: tendencias, claves y miradas. Madrid: Fundación Telefónica y Fundación Encuentro.

Sabella, R. A., Patchin, J. W., \& Hinduja, S. (2013). Cyberbullying myths and realities. Computers in Human Behavior, 29, 2703-2711. Recuperado de http://www.pleasval. 
k12.ia.us/juniorhigh/teachers/duncanmike/pdf/Health/myths\%20facts\%20about\%20 bullying.pdf.

Sasson, H., \& Mesch, G. (2014). Parental Mediation, Peer Norms and Risky Online Behavior. Computers in Human Behavior, 33, 32-38. doi: 10.1016/j.chb.2013.12.025

Save the children (2014). Pautas para padres y madres ante el acoso escolar. Recuperado de https://www.savethechildren.es/donde/espana/violencia-contra-la-infancia/acosoescolar-bullying

Smith, P. K., Mahdavi, J., Carvalho, M., Fisher, S., Russell, S., \& Tippett, N. (2008). Cyberbullying: Its nature and impact in secondary school pupils. Journal of Child Psychology and Psychiatry, 49, 376-385. doi: 10.1111/j.1469-7610.2007.01846.x

Stornaiuolo, A., DiZio, J. K., \& Hellmich, E. A. (2012). Desarrollando la comunidad: jóvenes, redes sociales y escuelas. Comunicar, 40(XX), 79-88. doi: 10.3916/C40-2013-02-08

Tejedor, S., \& Pulido, C., (2012). Retos y riesgos del uso de Internet por parte de los menores. ¿Cómo empoderarlos? Comunicar, 39(XX), 65-72. doi: 10.3916/C39-201202-06

Valkenburg, P. M., \& Peter, J. (2010). Online Communication among adolescents: An integrated model of its attraction, opportunities, and risks. Journal of Adolescent Health, 48, 121-127. doi: 10.1016/j.jadohealth.2010.08.020

Fecha de recepción: 14 de Febrero de 2016

Fecha de revisión: 14 de Febrero de 2016

Fecha de aceptación: 07 de Junio de 2016 
